\title{
Intramolekulare Alkoxycyanierungen und Alkoxyacylierungen: Neue Alkendifunktionalisierungen für den Aufbau von Sauerstoffheterocyclen
}

\author{
John P. Wolfe*
}

Alkene $\cdot$ Homogene Katalyse $\cdot$ Heterocyclen $\cdot$ Ketone .

Nitrile

$c$ drofurane und Dihydrobenzofurane, stellen wichtige Strukturbausteine bei einer Vielzahl von biologisch aktiven Verbindungen dar, einschließlich Naturstoffe und pharmazeutische Zielverbindungen. Somit besteht ein großes Interesse an der Entwicklung von neuen Methoden für den Aufbau dieser wichtigen Strukturen. ${ }^{[1]}$ Viele althergebrachte Verfahren beruhen auf der Ringbildung durch intramolekulare $\mathrm{S}_{\mathrm{N}} 2-\mathrm{Re}-$ aktionen und verwandte Strategien. Jedoch führen diese Ansätze typischerweise zur Bildung von nur einer Bindung während des Ringschlusses und erfordern zudem oft recht komplizierte Substrate. ${ }^{[1]}$

Die durch späte Übergangsmetalle katalysierten Alkencarboalkoxylierungen, eine Unterklasse der Alkendifunktionalisierungen ${ }^{[2]}$ sind für den Aufbau von Tetrahydrofuranen, Dihydrobenzofuranen und verwandten Sauerstoffheterocyclen außerordentlich hilfreich. Diese Reaktionen bewirken während des Ringschlusses die Bildung einer C-O-Bindung sowie einer dem Ring benachbarten C-C-Bindung und ferner den Aufbau von bis zu zwei Stereozentren (Schema 1). In den

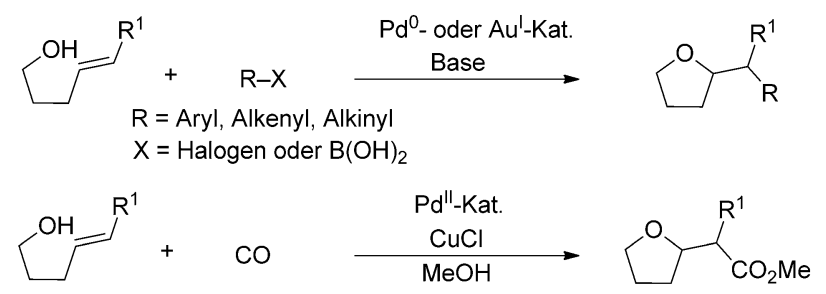

Schema 1. Metallkatalysierte Alkencarboalkoxylierungen von ungesättigten Alkoholen mit exogenen Kohlenstoffelektrophilen.

meisten Fällen umfassen diese Umwandlungen die Kupplung eines Alkohols, der ein angehängtes Alken trägt, an ein exogenes Kohlenstoffelektrophil, zum Beispiel ein Aryl-, Alkenyl- oder Alkinylhalogenid. ${ }^{[3,4]}$ Durch die Verwendung

[*] Prof. Dr. J. P. Wolfe

Department of Chemistry, University of Michigan

930 N. University Ave, Ann Arbor, MI 48109-1055 (USA)

E-Mail: jpwolfe@umich.edu von $\mathrm{CO}$ als Kohlenstoffelektrophil gelang auch die Einführung einer Esterfunktion. ${ }^{[5]}$

Kürzlich haben die Gruppen um Douglas und Nakao unabhängig voneinander zwei sich gegenseitig ergänzende neue Strategien für metallkatalysierte Alkencarboalkoxylierungen entwickelt, bei denen kein exogenes Kohlenstoffelektrophil erforderlich ist. ${ }^{[6,7]}$ Stattdessen ist das Kohlenstoffelektrophil kovalent an das cyclisierende Sauerstoffatom im Substrat gebunden, und die Carboalkoxylierungen werden durch die Aktivierung dieser C-O-Bindung mithilfe des Katalysators realisiert. Diese beiden Ansätze führen zur Bildung von Dihydrobenzofuranderivaten mit funktionellen Gruppen (Ketone oder Nitrile), die für den weiteren Aufbau molekularer Zielverbindungen sehr nützlich sind und über die zuvor entwickelten Alkencarboalkoxylierungsmethoden nicht direkt eingeführt werden konnten.

Bei der Methode der Gruppe um Douglas wird ein kationischer $\mathrm{Rh}^{\mathrm{I}}$-Komplex verwendet, um die intramolekulare Alkoxyacyclierung der acylierten 2-Allylphenol-Derivate zu katalysieren (Schema 2) ${ }^{[6]}$ Diese Reaktionen liefern die 2-

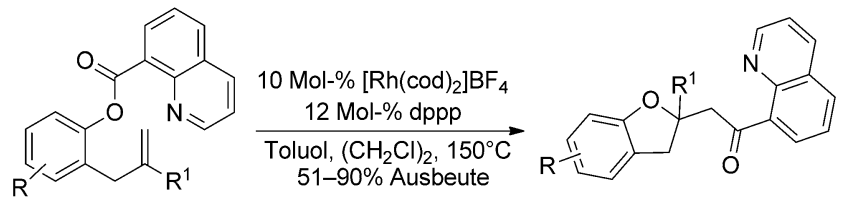

Schema 2. Rhodium-katalysierte intramolekulare Alkenalkoxyacyclierungen von Douglas. cod = 1,5-Cyclooctadien, dppp = Propan-1,3-diylbis(diphenylphosphan).

Acylmethyldihydrobenzofuran-Derivate mit guten Ausbeuten $(51-90 \%)$ und sind auch für die Bildung von analogen Chroman- und Benzodioxin-Produkten anwendbar (wobei die Ausbeute an Benzodioxin allerdings nur mäßig ist). $\mathrm{Zu}$ diesem Zeitpunkt muss jedoch für die erfolgreiche Erzeugung des Produktes eine Chinolinfunktion als lenkende Gruppe an der Acyleinheit des Substrats vorhanden sein. Diese Gruppe hilft, konkurrierende Decarbonylierungen von intermediären Acylrhodiumspezies $\mathrm{zu}$ vermeiden und könnte auch eine Rolle bei der Katalysatorbindung zur Steuerung der C-OBindungsaktivierung spielen. 


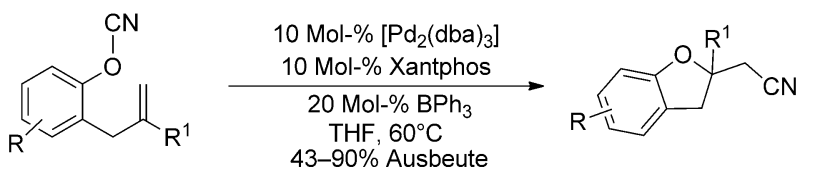

Schema 3. Palladium-katalysierte intramolekulare Alkenalkoxycyanierungen von Nakao. dba=trans, trans-Dibenzylidenaceton.

Die Gruppe um Nakao wendete eine verwandte Strategie zur Erzeugung von 2-Cyanmethyldihydrobenzofuran-Derivaten an (Schema 3). In diesem Fall wurden von 2-Allylphenol abgeleitete Cyanate als Substrate verwendet und die intramolekularen Alkoxycyanierungen wurden mithilfe eines dualen Katalysatorsystems, bestehend aus $\left[\mathrm{Pd}_{2}(\mathrm{dba})_{3}\right] /$ Xantphos $(10 \mathrm{Mol}-\%)$ und $\mathrm{BPh}_{3}(20 \mathrm{Mol}-\%)$, realisiert. ${ }^{[7]}$ Der Palladiumkatalysator ist an den entscheidenden bindungsbildenden Schritten beteiligt, und die Lewis-Säure $\mathrm{BPh}_{3}$ wird an das Nitril gebunden und erleichtert somit die Insertion des Palladiumkomplexes in die O-CN-Bindung der Cyanatgruppe. Die Produkte wurden mit mäßiger bis guter Ausbeute erhalten, und die Autoren zeigten, dass sowohl Fünf- als auch Sechsringe eingeführt werden können. Die Reaktionsbedingungen sind so mild, dass Estergruppen durchaus vorhanden sein können, und Halogensubstituenten $(\mathrm{F}, \mathrm{Cl}, \mathrm{Br})$ am aromatischen Ring werden während dieser Umwandlungen auch nicht abgespalten. Jedoch sind die Ausbeuten für die Reaktionen dieser funktionalisierten Substrate (43-79\%) etwas geringer als für nichtfunktionalisierte Derivate (60-90\%).

Die intramolekularen Alkoxyacyclierungen und Alkoxycyanierungen, die von Douglas bzw. Nakao beschrieben wurden, scheinen über ähnliche mechanistische Wege abzulaufen (Schema 4). In beiden Fällen findet eine oxidative

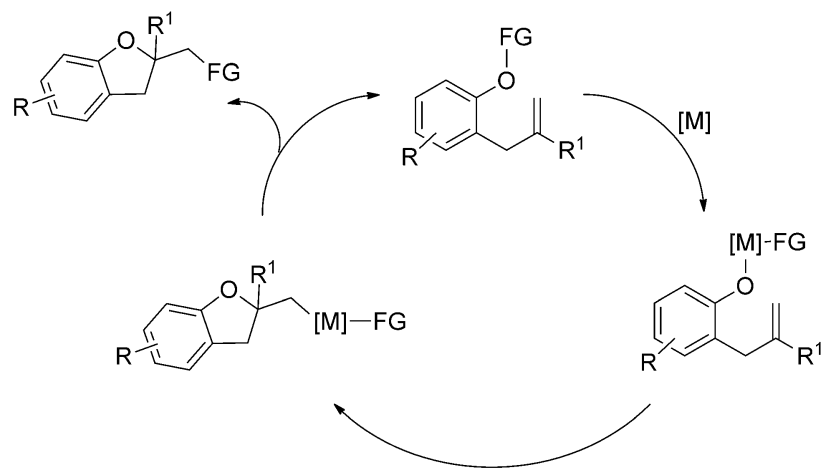

Schema 4. Mechanismus der intramolekularen Alkenalkoxyfunktionalisierungen. $\mathrm{FG}=$ funktionelle Gruppe, $\mathrm{M}=$ Metall

Addition der O-FG-Bindung an den niedervalenten Metallkatalysator statt, die einen intermediären Metall-AlkoxidKomplex erzeugt. ${ }^{[8]}$ Bei der Insertion des Alkens in die M-OBindung ${ }^{[4 a, b, 9]}$ und der anschließenden reduktiven Eliminierung unter C-FG-Bindungsbildung werden dann die funktionalisierten heterocyclischen Produkte erhalten. Dieser Mechanismus ist verwandt mit dem anderer Carboalkoxylierungen, die durch späte Übergangsmetalle katalysiert werden, ${ }^{[4]}$ unterscheidet sich jedoch hinsichtlich des Weges, auf dem das entscheidende Metall-Alkoxid-Intermediat gebildet wird.

Wie bei allen neuen Synthesemethoden sind die Möglichkeiten dieser neuen Umwandlungen noch nicht vollständig erforscht worden. Im Unterschied zu anderen durch späte Übergangsmetalle katalysierten Carboalkoxylierungen sind die neuen Alkoxyfunktionalisierungen gegenwärtig noch auf den Aufbau von Heterocyclen mit anellierten Arylringen beschränkt. Außerdem ist eine Substitution am internen Alkenkohlenstoffatom erforderlich, um konkurrierende $\beta$-Hydrid-Eliminierungen zu vermeiden. Des Weiteren werden bei zukünftigen Untersuchungen sicher auch diastereo- und enantioselektive Varianten dieser Reaktionen im Fokus stehen. Nichtsdestotrotz stellen die neuen von Douglas und Nakao entwickelten intramolekularen Alkenalkoxyfunktionalisierungen einen bedeutenden konzeptionellen Fortschritt auf diesem Gebiet dar. Mit diesen Umwandlungen ist die Einführung von Keto- oder Cyangruppen in heterocyclische Verbindungen möglich. Sie laufen unter relativ milden Bedingungen $a b$, da kein Basenzusatz notwendig ist. Die Strategie für die Bildung von Heterocyclen durch eine metallkatalysierte Alkeninsertion in die Bindung einer funktionellen Gruppe, die ein Heteroatom enthält, verfügt über ein großes Nutzungspotenzial, und es sind viele Anwendungsmöglichkeiten in der Entwicklung von Reaktionen und der Synthese von komplexen molekularen Verbindungen vorstellbar.

Eingegangen am 8. Juni 2012

Online veröffentlicht am 5. September 2012

[1] a) J. P. Wolfe, M. B. Hay, Tetrahedron 2007, 63, 261-290; b) F. Bertolini, M. Pineschi, Org. Prep. Proc. Int. 2009, 41, 385-418.

[2] K. H. Jensen, M. S. Sigman, Org. Biomol. Chem. 2008, 6, $4083-$ 4088.

[3] Übersichten: a) R. I. McDonald, G. Liu, S. S. Stahl, Chem. Rev. 2011, 111, 2981-3019; b) J. P. Wolfe, Synlett 2008, 2913-2937.

[4] a) J. P. Wolfe, M. A. Rossi, J. Am. Chem. Soc. 2004, 126, $1620-$ 1621; b) M. B. Hay, J. P. Wolfe, J. Am. Chem. Soc. 2005, 127, $16468-16476$; c) S. Hayashi, H. Yorimitsu, K. Oshima, J. Am. Chem. Soc. 2009, 131, 2052-2053; d) G. Zhang, L. Cui, Y. Wang, L. Zhang, J. Am. Chem. Soc. 2010, 132, 1474-1475; e) S. Nicolai, J. Waser, Org. Lett. 2011, 13, 6324-6327.

[5] a) M. F. Semmelhack, C. Bodurow, J. Am. Chem. Soc. 1984, 106, 1496-1498; b) M. F. Semmelhack, N. Zhang, J. Org. Chem. 1989, 54, 4483-4485.

[6] G. T. Hoang, V. J. Reddy, H. H. K. Nguyen, C. J. Douglas, Angew. Chem. 2011, 123, 1922-1924; Angew. Chem. Int. Ed. 2011, 50, $1882-1884$.

[7] D. C. Koester, M. Kobayashi, D. B. Werz, Y. Nakao, J. Am. Chem. Soc. 2012, 134, 6544-6547.

[8] a) N. Chatani, H. Tatamidani, Y. Ie, F. Kakiuchi, S. Murai, J. Am. Chem. Soc. 2001, 123, 4849-4850; b) T. Yamamoto, S. Miyashita, Y. Naito, S. Komiya, T. Ito, A. Yamamoto, Organometallics 1982, 1, 808-812; c) K. Fukumoto, A. A. Dahy, T. Oya, K. Hayasaka, M. Itazaki, N. Koga, H. Nakazawa, Organometallics 2012, 31, $787-790$.

[9] a) T. Hayashi, K. Yamasaki, M. Mimura, Y. Uozumi, J. Am. Chem. Soc. 2004, 126, 3036-3037; b) R. M. Trend, Y. K. Ramtohul, B. M. Stoltz, J. Am. Chem. Soc. 2005, 127, 17778-17788; c) P. Zhao, C. D. Incarvito, J. F. Hartwig, J. Am. Chem. Soc. 2006, 128, 9642-9643. 